\title{
DENSITY OF ZEROS OF SOME ORTHOGONAL POLYNOMIALS
}

\author{
Yang Chen and Nigel Lawrence
}

\begin{abstract}
In this paper, we study the asymptotic eigenvalue density of large $n \times n$ random Hermitian matrices. The eigenvalue density can be interpreted in the context of orthogonal polynomials as the density of zeros. We adopt two approaches; the first, using a recent theorem, gives the density of zeros as an integral representation with the (appropriately scaled) recurrence coefficients as input. The second makes use of the Coulomb fluid approach pioneered by Dyson where the weight with respect to which the polynomials are orthogonal is the input.

The zero density of the Stieltjes-Wigert, $q^{-1}$-Hermite, $q$-Laguerre polynomials and a constructed set of orthogonal polynomials are obtained. In the last two cases, the density can be expressed in terms of complete and incomplete elliptic integrals of various kinds.

We also compute, in some cases, the effective potentials from the densities.
\end{abstract}

\section{Introduction}

In the application of the theory of $n \times n$ random matrices, a quantity of central interest is the asymptotic eigenvalue density for large $n$. If the matrix is complex Hermitian, it can be shown that the eigenvalue density is the density of zeros of certain polynomials orthogonal with respect to a positive weight supported on $\mathbf{R}$ or a subset of $\mathbf{R}$ where $n$ is the degree.

A more conventional technique for computing the asymptotic density is based on potential theory, and in the physical context, the Coulomb fluid method pioneered by Dyson in the 1960s [7]. For an excellent and up-to-date book on potential theoretic methods, see [11]. For a physical approach to the density, see [4]. This latter technique relies on the weight function, $w(x)$, and more precisely, the external potential associated with it, $v(x):=-\ln w(x)$. However, if the external potential increases sufficiently slowly near infinity, such as those of the $q^{-1}$-Hermite and the $q$-Laguerre polynomials where $v(x)=\mathrm{O}\left([\ln |x|]^{2}\right)$, the associated classical moment problem is indeterminate $[1]$.

We would like to emphasize that the long tail seen in the equilibrium densities in all but the last example studied in this paper is due to the fact that the zeros of these polynomials are widely separated, as a consequence of which the density behaves like $O(1 / x)$ as $x \rightarrow \infty$.

These are of particular interest in the theory of quantum transport in disordered systems [5] because, in such examples, physical considerations suggest that $v(x)=$ $\mathrm{O}\left([\ln x]^{2}\right)$. It is important therefore to have an independent tool to compute the zero

Received 5 February 1998, revised 20 June 1998.

1991 Mathematics Subject Classification: 35D45, 30E05.

Key words and phrases: eigenvalue density, orthogonal polynomials, Coulomb fluid. 
density as this depends only on the moments of the weight function and not on the weight function itself. We note here that the zero density plays an important role in determining the strong asymptotics of orthogonal polynomials and these in turn are essential ingredients in determining fundamental physical quantities such as the gap formation probability $[4,6]$.

Recently, Kuijlaars and Van Assche [10] proved that under some mild conditions imposed on the (varying) recurrence coefficients, the zero density can be computed by quadrature. In this situation, the input consisting of the recurrence coefficients therefore is independent of the weight.

The purpose of this paper is to show how the asymptotic zero density of a set of orthogonal polynomials may be obtained from the recurrence relation. This proves to be a complementary tool for obtaining the density and provides an independent check on the Coulomb fluid technique. We consider various examples of orthogonal polynomials with varying recurrence coefficients, and then, utilizing a theorem given in [10], stated below, the density is derived. In the case of Stieltjes-Wigert polynomials, we show that the density calculated by this method is identical to that found by the Coulomb fluid approach. Considering the $q$-Laguerre polynomials where the parameter $\gamma$ is assumed to vary with polynomial degree, we illustrate how, in a particular case, the density is expressed in terms of elliptic functions. This is also the motivation behind the inclusion of the constructed example, where an algebraically simple model has a density given in terms of elliptic functions. These are surprising as there are few examples in which the equilibrium densities are expressed in terms of higher transcendental functions.

This paper is organized as follows. In $\S 2$, the zero density of the Stieltjes-Wigert polynomials is obtained via two methods: the Theorem stated below and the potential theoretic/Coulomb fluid method. $\S 3$ is concerned with the zero density of the $q^{-1}$-Hermite polynomials. In $\S 4$, the zero density of the $q$-Laguerre polynomials is given for two cases: in the first, the index $\gamma$ is independent of $n$, the polynomial degree, and, in the second, $\gamma$ varies with $n$. To show that the appearance of the elliptic functions in $\S 4$ is quite natural, we include in $\S 5$ a constructed set of orthogonal polynomials. Figures which graph the zero density are included.

Theorem 1.1. [10] For each natural number $N$, let two sequences $\left\{\alpha_{n, N}\right\}_{n=0}^{\infty}$ and $\left\{\beta_{n, N}\right\}_{n=0}^{\infty}$, with $\beta_{n, N}>0$, of recurrence coefficients be given, along with the orthogonal polynomials $p_{n, N}(x)$ which are generated by the recurrence relation

$$
x p_{n, N}(x)=p_{n+1, N}(x)+\alpha_{n, N} p_{n, N}(x)+\beta_{n, N} p_{n-1, N}(x), \quad n \geq 0,
$$

and the initial conditions $p_{0, N}(x) \equiv 1$ and $p_{-1, N}(x) \equiv 0$. Assume that $n / N \rightarrow t>0$ as $n \rightarrow \infty$ and $N \rightarrow \infty$. Assume there exist two continuous functions $\alpha:(0, \infty) \rightarrow \mathbf{R}$, $\beta:(0, \infty) \rightarrow[0, \infty)$, such that

$$
\lim _{n / N \rightarrow t} \alpha_{n, N}=\alpha(t), \quad \lim _{n / N \rightarrow t} \beta_{n, N}=\beta(t),
$$

whenever $t>0$. Defining the functions

$$
a(t):=\alpha(t)-2 \sqrt{\beta(t)}, \quad b(t):=\alpha(t)+2 \sqrt{\beta(t)}, \quad t>0 .
$$

We then have, for every $t>0$, the asymptotic zero density given by

$$
\sigma(x, t)=\frac{1}{\pi t} \int_{t^{-}(x)}^{\min \left(t, t^{+}(x)\right)} \frac{d s}{\sqrt{(b(s)-x)(x-a(s))}}
$$


where $t^{-}(x)$ and $t^{+}(x)$ are the end points of the interval, defined for each $x$ by the set $\{s>0: a(s) \leq x \leq b(s)\}$.

Note that $p_{n, N}(x)$ defined above is monic and (1.3) was derived as thermodynamic relations of Hermitian random matrix models [4]. Although (1.4) is proved by starting from the recurrence relations [10], we give below a heuristic justification of how it can be obtained using the Coulomb fluid approach. In the potential theoretic/Coulomb fluid approach, with varying weights, that is $v(x) \rightarrow N v(x)$ where $0 \leq N<\infty$, the zero density, denoted by $\sigma(x, t)$, satisfies the following integral equation

$$
v(x)-2 t \int_{a(t)}^{b(t)} \sigma(y, t) \ln |x-y| d y=A(t)=\text { constant, } \quad x \in[a(t), b(t)],
$$

where

$$
\int_{a(t)}^{b(t)} \sigma(x, t) d x=1
$$

By taking a derivative with respect to $x$, the above integral equation becomes

$$
v^{\prime}(x)=2 P \int_{a(t)}^{b(t)} \frac{t \sigma(y, t)}{x-y} d y, \quad x \in[a(t), b(t)] .
$$

If $f(x, t):=\partial_{t}[t \sigma(x, t)]$, then

$$
\int_{a(t)}^{b(t)} f(x, t) d x=1
$$

Noting that $\sigma(a(t), t)=\sigma(b(t), t)=0, f(x, t)$ is seen to satisfy

$$
P \int_{a(t)}^{b(t)} \frac{f(y, t)}{x-y} d y=0, \quad x \in[a(t), b(t)]
$$

and the unique solution is

$$
\frac{\partial t \sigma(x, t)}{\partial t}=\frac{1}{\pi \sqrt{(b(t)-x)(x-a(t))}} .
$$

An integration gives (1.4). This derivation again is based on the thermodynamic approach of [4]. The above argument does not replace the original proof of the theorem [10]. Regarding the accuracy of the Coulomb fluid approach, we have the following quote:

"These assumptions ... can be summarized in the single statement that for large $N$ the Coulomb gas obeys the laws of classical thermodynamics. The assumption ... means that the free energy at any point [is] a function of the local density and temperature alone. To a physicist these assumptions are so hallowed by custom that they hardly require justification. Every application of thermodynamics to systems of strongly interacting atoms or molecules rests on assumptions of this kind." [7, page 158]. 


\section{The Stieltjes-Wigert polynomials}

For the monic Stieltjes-Wigert polynomials, the recurrence coefficients are

$$
\begin{aligned}
& \alpha_{n}=q^{-2 n}\left[q^{-3 / 2}(1+q)-q^{n-1 / 2}\right], \\
& \beta_{n}=q^{-4 n}\left(1-q^{n}\right)
\end{aligned}
$$

where $0<q<1$. Note that the moment problem is indeterminate. We now introduce a scaling in the parameter $q$ described below; this corresponds to having varying recurrence coefficients. The scaling introduces an additional parameter independent of the polynomial degree $n$. This also is equivalent to a varying weight. The introduction of the additional scaling parameter does not alter the long tail in the equilibrium density.

Suppose $q=\exp [-1 / N], N>0$. Thus, in the limits $n \rightarrow \infty$ and $N \rightarrow \infty$ with $t=n / N$, we find

$$
\alpha_{n, N} \rightarrow \alpha(t)=2 \mathrm{e}^{2 t}-\mathrm{e}^{t}, \quad t>0, \quad \beta_{n, N} \rightarrow \beta(t)=\mathrm{e}^{4 t}\left(1-\mathrm{e}^{-t}\right), \quad t>0,
$$

and

$$
a(t)=2 \mathrm{e}^{2 t}\left(1-\frac{\mathrm{e}^{-t}}{2}-\sqrt{1-\mathrm{e}^{-t}}\right), \quad b(t)=2 \mathrm{e}^{2 t}\left(1-\frac{\mathrm{e}^{-t}}{2}+\sqrt{1-\mathrm{e}^{-t}}\right) .
$$

The zero density, $\sigma(x ; t)$, according to $(1.4)$, is

$$
\sigma(x ; t)=\frac{1}{\pi t} \int_{t^{-}(x)}^{t} \frac{d s}{\sqrt{(b(s)-x)(x-a(s))}}
$$

where $t^{-}(x)$ satisfies

$$
a\left(t^{-}\right)=x \quad \text { or } \quad b\left(t^{-}\right)=x .
$$

A simple integration gives

$$
\sigma(x ; t)=\frac{1}{\pi x t} \tan ^{-1} \frac{\sqrt{(b(t)-x)(x-a(t))}}{x+\sqrt{a(t) b(t)}}, \quad a(t) \leq x \leq b(t) .
$$

Note that $a(t)$ tends to $1 / 4$ with exponentially fast speed and the smallest zero is $\sim 1 / 4$. Intuitively, we may expect $a(t) \rightarrow 0$ as $t \rightarrow \infty$ in the Coulomb fluid picture; however, as the potential only increases slowly as $x$ increases, $\lim _{t \rightarrow \infty} a(t) \neq 0$.

According to the integral equation of the Coulomb fluid theory [4], the (effective) potential generated by the density is

$$
\frac{v^{\prime}(x)}{2}=t P \int_{a}^{b} \frac{\sigma(y, t)}{x-y} d y, \quad x \in[a, b],
$$

where the $t$ dependence of $a$ and $b$ are not displayed. Thus, using (2.7),

$$
\begin{aligned}
\frac{v^{\prime}(x)}{2} & =\frac{1}{\pi} P \int_{a}^{b} \tan ^{-1}\left(\frac{\sqrt{(b-y)(y-a)}}{y+\sqrt{a b}}\right) \frac{d y}{y(x-y)} \\
& =\frac{t}{x}+\frac{1}{\pi x} P \int_{a}^{b} \tan ^{-1}\left(\frac{\sqrt{(b-y)(y-a)}}{y+\sqrt{a b}}\right) \frac{d y}{x-y} .
\end{aligned}
$$

To evaluate the above integral, we make use of the Plemelj-Sokhotski lemma [8]. Consider

$$
J(x):=\int_{a}^{b} \tan ^{-1}\left(\frac{\sqrt{(b-y)(y-a)}}{y+\sqrt{a b}}\right) \frac{d y}{x-y}, \quad x>b
$$


Integration by parts produces a logarithm and using the identity

$$
\ln (A+B)=\ln A+\int_{0}^{1} \frac{B d \lambda}{A+\lambda B}
$$

we find after some computation:

$$
J(x)=\pi \ln \left(\frac{2}{\sqrt{a}+\sqrt{b}} \cdot \frac{\sqrt{a(x-b)}+\sqrt{b(x-a)}}{\sqrt{x-b}+\sqrt{x-a}}\right), \quad x>b .
$$

Therefore, the principal value integral is obtained through the analytic continuation of $x$ to $x \in[a, b]$ and Plemelj-Sokhotski lemma. Thus,

$$
\Re J(x)=\pi \ln \left(\frac{2 \sqrt{x}}{\sqrt{b}+\sqrt{a}}\right)=\pi \ln \left(\frac{\sqrt{x}}{\sqrt{a b}}\right)
$$

and

$$
v^{\prime}(x)=\frac{\ln x}{x}
$$

and with $q=\exp [-1 / N]$. Therefore, the effective potential is

$$
v(x)=\frac{(\ln x)^{2}}{2}, \quad 0 \leq x<\infty .
$$

We now apply the Coulomb fluid technique. From the weight

$$
w(x)=\exp \left[-\frac{(\ln x)^{2}}{2}\right], \quad 0 \leq x<\infty,
$$

the potential reads $v(x)=\frac{(\ln x)^{2}}{2}$. The solution of the integral equation is

$$
\begin{aligned}
\sigma(x) & =\frac{\sqrt{(b-x)(x-a)}}{2 \pi^{2} t} P \int_{a}^{b} \frac{\ln y}{y \sqrt{(b-y)(y-a)}} \frac{d y}{y-x} \\
& =\frac{1}{\pi x t} \tan ^{-1}\left[\frac{(\sqrt{b}-\sqrt{a}) \sqrt{(b-x)(x-a)}}{\sqrt{a}(b-x)+\sqrt{b}(x-a)}\right], \quad x \in[a, b],
\end{aligned}
$$

found to be in agreement with the density obtained from the Theorem. Here $a$ and $b$ are determined by the normalization condition on the density and a supplementary condition [4] identical to (2.4). The zero density of the Stieltjes-Wigert polynomials is graphed in Figure 1 for various values of $t$.

\section{The $q^{-1}$-Hermite polynomials}

The $q^{-1}$-Hermite polynomials satisfy

$$
2 x h_{n}(x \mid q)=h_{n+1}(x \mid q)+\frac{1-q^{n}}{q^{n}} h_{n-1}(x \mid q), \quad 0 \leq q<1 .
$$

If we denote $h_{n}(x / 2 \mid q)$ by $p_{n}(x)$, then

$$
x p_{n}(x)=p_{n+1}(x)+\frac{1-q^{n}}{q^{n}} p_{n-1}(x) .
$$

Thus, $\beta_{n}=\left(1-q^{n}\right) / q^{n}$. If $q=e^{-1 / N}, N>0$, and $n / N=t$, then

$$
\beta(t)=\frac{1-e^{-t}}{e^{-t}}=e^{t}-1, \quad b^{2}(t)=4\left(e^{t}-1\right) .
$$




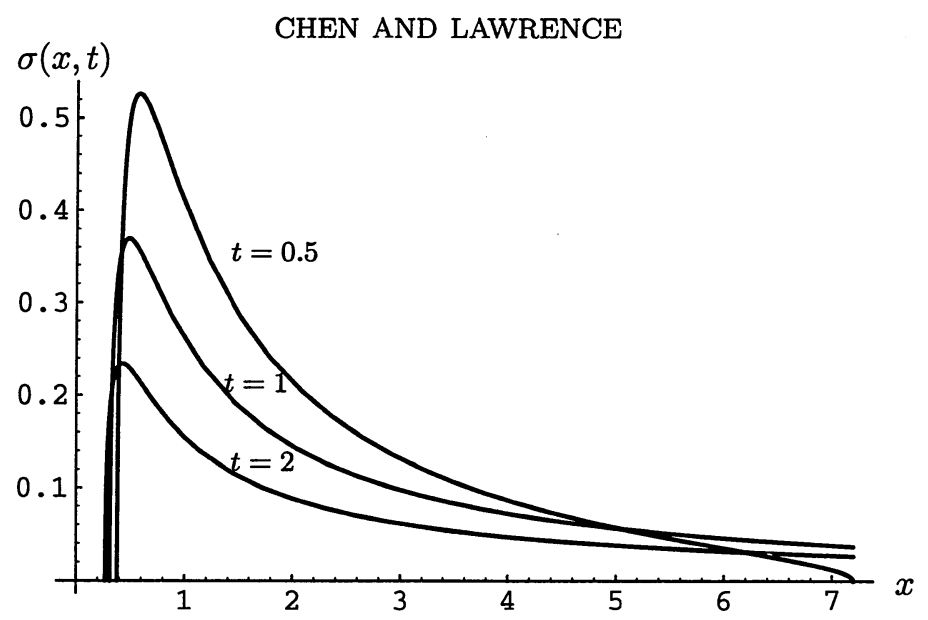

Figure 1. Zero density of the Stieltjes-Wigert polynomials. Plotted here are the densities for the cases where $t=0.5,1,2$. Note as $t$ increases, $a(t) \rightarrow 1 / 4$.

The density is

$$
\sigma(x ; t)=\frac{1}{\pi t} \int_{t^{-}(x)}^{t} \frac{d s}{\sqrt{b^{2}(s)-x^{2}}}
$$

where

$$
e^{t^{-}}=1+\left(\frac{x}{4}\right)^{2}
$$

Thus,

$$
\sigma(x ; t)=\frac{1}{\pi t} \frac{2}{\sqrt{4+x^{2}}} \tan ^{-1}\left(\frac{\sqrt{b^{2}-x^{2}}}{\sqrt{4+x^{2}}}\right) .
$$

We compute here the effective potential. Note that since $\sigma(x, t)$ is even in $x$,

$$
\frac{v^{\prime}(x)}{2}=2 x t P \int_{0}^{b} \frac{\sigma(y, t)}{x^{2}-y^{2}} d y \text {. }
$$

Note also that

$$
\sigma(\sqrt{s}, t)=\frac{2}{\pi t} \int_{0}^{1} \frac{\left(b^{2}-s\right) d \mu}{\left(b^{2}-s\right) \mu^{2}+(4+s)}, \quad 0 \leq s \leq b^{2}(t) .
$$

After some elementary calculations,

$$
\frac{d v}{d x^{2}}=\frac{1}{\sqrt{4 x^{2}+x^{4}}} \ln \left[\frac{2}{x^{2}+2-\sqrt{4 x^{2}+x^{4}}}\right] .
$$

Thus,

$$
v(x)=\frac{1}{2}\left[\ln \left(\frac{2+x^{2}-\sqrt{4 x^{2}+x^{4}}}{2}\right)\right]^{2}+\text { constant. }
$$

The graph of the zero density of the $q^{-1}$-Hermite polynomials is given in Figure 2 for various values of $t$. 


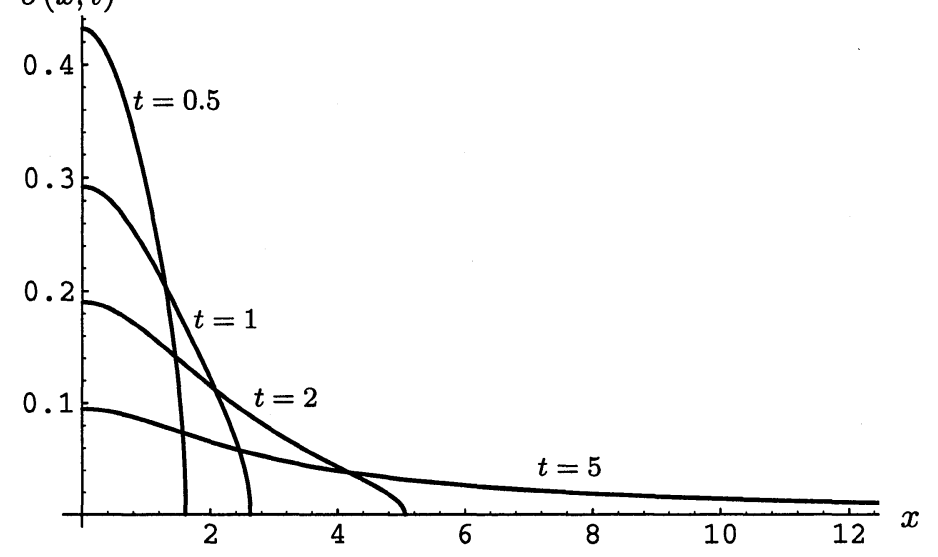

FIgURE 2. Zero density of the $q^{-1}$-Hermite polynomials. Contrasted here are the densities for $t=0.5,1,2,5$

\section{The $q$-Laguerre polynomials}

In this and the next sections, the density is expressed in terms of elliptic functions. For future reference, we follow the definitions given in [9].

An elliptic function of the first kind is given by

$$
F(\psi, k):=\int_{0}^{\sin \psi} \frac{d x}{\sqrt{\left(1-x^{2}\right)\left(1-k^{2} x^{2}\right)}}, \quad k^{2}<1 .
$$

An elliptic function of the third kind is given by

$$
\Pi(\psi, n, k):=\int_{0}^{\sin \psi} \frac{d x}{\left(1-n x^{2}\right) \sqrt{\left(1-x^{2}\right)\left(1-k^{2} x^{2}\right)}}, \quad k^{2}<1 .
$$

If $\psi=\pi / 2$, the elliptic function is said to be complete.

The monic $q$-Laguerre polynomials satisfy the following three term recurrence relation

$$
x L_{n}^{(\gamma)}(x ; q)=L_{n+1}^{(\gamma)}(x ; q)+\alpha_{n} L_{n}^{(\gamma)}(x ; q)+\beta_{n} L_{n-1}^{(\gamma)}(x ; q), \quad \gamma \in[0,1),
$$

where

$$
\begin{aligned}
& \alpha_{n}=\frac{q\left(1-q^{n}\right)+\left(1-q^{n+\gamma+1}\right)}{q^{2 n+\gamma+1}(1-q)} \\
& \beta_{n}=\frac{\left(1-q^{n+\gamma}\right)\left(1-q^{n}\right)}{q^{4 n+2 \gamma-1}(1-q)^{2}} .
\end{aligned}
$$

As in the previous section, $q=\exp [-1 / N]$ which tends to 1 if $N \rightarrow \infty$. To remove the problem of having $(1-q)$ in the denominator of the recurrence coefficients, multiply throughout the recurrence relation by $(1-q)$. Thus, with $y:=(1-q) x$ and

$$
p_{n}(y):=(1-q)^{n} L_{n}^{(\gamma)}\left(\frac{y}{1-q} ; q\right)
$$

$p_{n}(y)$ satisfies the monic recurrence relation

$$
y p_{n}(y)=p_{n+1}(y)+\alpha_{n} p_{n}(y)+\beta_{n} p_{n-1}(y)
$$


where

$$
\begin{aligned}
& \alpha_{n}=\frac{\left(1-q^{n}\right) q+\left(1-q^{n+\gamma+1}\right)}{q^{2 n+\gamma+1}}, \\
& \beta_{n}=\frac{\left(1-q^{n+\gamma}\right)\left(1-q^{n}\right)}{q^{4 n+2 \gamma-1}} .
\end{aligned}
$$

With $q:=\exp [-1 / N], N>0, n / N=t$, and $\gamma$ fixed independent of $n$, we find

$$
\begin{aligned}
& \alpha_{n, N} \rightarrow \alpha(t)=2 e^{t}\left(e^{t}-1\right), \\
& \beta_{n, N} \rightarrow \beta(t)=e^{2 t}\left(e^{t}-1\right)^{2},
\end{aligned}
$$

and

$$
a(t):=\alpha(t)-2 \sqrt{\beta(t)}=0 \quad \text { and } \quad b(t):=\alpha(t)+2 \sqrt{\beta(t)}=4 e^{t}\left(e^{t}-1\right) .
$$

The zero density is

$$
\sigma(x, t)=\frac{1}{\pi t} \int_{t^{-}}^{t} \frac{d s}{\sqrt{x(b(s)-x)}} \quad \text { where } \quad b\left(t^{-}\right)=x .
$$

An elementary integration then gives

$$
\sigma(x, t)=\frac{1}{\pi t x} \tan ^{-1}\left[\frac{\sqrt{x[b(t)-x]}}{x+2 e^{t}}\right], \quad x \in[0, b(t)] .
$$

We now proceed by evaluating the effective potential, again using (2.8). We find

$$
\begin{aligned}
\frac{v^{\prime}(x)}{2} & =\frac{1}{\pi} P \int_{0}^{b} \frac{1}{(x-y) y} \tan ^{-1}\left[\frac{\sqrt{y(b-y)}}{y+1+\sqrt{1+b}}\right] d y \\
& =\frac{t}{x}+\frac{1}{\pi x} P \int_{0}^{b} \frac{1}{x-y} \tan ^{-1}\left[\frac{\sqrt{y(b-y)}}{y+1+\sqrt{1+b}}\right] d y
\end{aligned}
$$

where we have used the fact that $2 e^{t}=1+\sqrt{1+b}$ and the normalization condition of the density function. The principal value integral in (4.9) is evaluated by considering the integral

$$
I(x)=\int_{0}^{b} \frac{1}{x-y} \tan ^{-1} \frac{\sqrt{y(b-y)}}{y+1+\sqrt{1+b}} d y \quad x>b .
$$

The integral $I(x)$ can be simplified by an integration by parts and an application of (2.10), thus

$$
I(x)=\frac{\pi}{2}\left[\ln \frac{4(x+1)(\sqrt{1+b}-1)}{b(\sqrt{1+b}+1)}+\ln \frac{(\sqrt{x}-\sqrt{x-b})(\sqrt{(1+b) x}+\sqrt{x-b})}{(\sqrt{x}+\sqrt{x-b})(\sqrt{(1+b) x}-\sqrt{x-b})}\right] .
$$

Now an analytic continuation to $x \rightarrow x \in[0, b]$ gives,

$$
I^{+}(x):=\lim _{\varepsilon \rightarrow 0} I(x+i \varepsilon)=\frac{\pi}{2} \ln \frac{4(x+1)(\sqrt{1+b}-1)}{b(\sqrt{1+b}+1)}-i \pi \tan ^{-1} \frac{\sqrt{x(b-x)}}{x+1+\sqrt{1+b}} .
$$

Applying the Plemelj-Sokhotski lemma, we obtain the principal value integral

$$
\begin{aligned}
v^{\prime}(x) & =\frac{2 t}{x}+\frac{1}{x} \ln \frac{4(x+1)(\sqrt{1+b}-1)}{b(\sqrt{1+b}+1)} \\
& =\frac{1}{x} \ln (x+1)
\end{aligned}
$$


where we again use the fact that $2 e^{t}=1+\sqrt{1+b}$.

There is an interesting case where $\gamma$ varies with $n$ :

$$
\gamma(n)=\varrho n \text {. }
$$

Recalling the recurrence relation coefficients, let $q=e^{-1 / N}, N>0$, and $n / N=t$. Thus,

$$
\begin{aligned}
\alpha_{n, N} \rightarrow \alpha(t) & =e^{(2+\varrho) t}\left[\left(1-e^{-t}\right)+\left(1-e^{-(1+\varrho) t}\right)\right], \\
\beta_{n, N} \rightarrow \beta(t) & =e^{2(2+\varrho) t}\left(1-e^{-(1+\varrho) t}\right)\left(1-e^{-t}\right) .
\end{aligned}
$$

Note that since $\beta(t)>0$, we must have $\varrho>-1$. Thus, $a(t)$ and $b(t)$ are

$$
\begin{aligned}
& a(t)=e^{(2+\varrho) t}\left(2-e^{-t}\left(1+e^{-\varrho t}\right)-2 \sqrt{\left(1-e^{-(1+\varrho) t}\right)\left(1-e^{-t}\right)}\right) \\
& b(t)=e^{(2+\varrho) t}\left(2-e^{-t}\left(1+e^{-\varrho t}\right)+2 \sqrt{\left(1-e^{-(1+\varrho) t}\right)\left(1-e^{-t}\right)}\right) .
\end{aligned}
$$

For $\varrho>-1$, it is not clear whether the density can be obtained in closed form. However, there is a special case where $\varrho=1$, and the asymptotic zero density can be evaluated explicitly. Thus,

$$
\sigma(x, t)=\frac{1}{\pi t} \int_{t^{-}(x)}^{\min \left(t^{+}(x), t\right)} \frac{d s}{\sqrt{(x-a(s))(b(s)-x)}} \text { where } b\left(t^{-}\right)=x \text { and } a\left(t^{+}\right)=x .
$$

After using the expressions for $a(t)$ and $b(t)$ and the substitution $\lambda=e^{s}$, we have

$$
\sigma(x, t)=\frac{1}{\pi t} \int_{e^{t^{-}}}^{\min \left(e^{t^{+}}, e^{t}\right)} \frac{d \lambda}{\lambda \sqrt{-\lambda^{4}+2(2 x+1) \lambda^{3}-(2 x+1) \lambda^{2}-2 x \lambda-x^{2}}} .
$$

Let us denote $P_{4}(\lambda):=\lambda^{4}-2(2 x+1) \lambda^{3}+(2 x+1) \lambda^{2}+2 x \lambda+x^{2}$, and note that $P_{4}\left(e^{t^{-}}\right)=P_{4}\left(e^{t^{+}}\right)=0$.

Since the integrand is a product of a rational function of $\lambda$ and the square root of a fourth degree polynomial in $\lambda$, the density may be expressed in terms of elliptic functions [3]. In order to determine the exact form, we must first study the nature of $P_{4}(\lambda)$, following [2]. Making the substitution $\lambda=z+(2 x+1) / 2$,

$$
P_{4}(z)=z^{4}+p(x) z^{2}+q(x) z+r(x)
$$

where

Now, for any $u$,

$$
\begin{aligned}
p(x) & :=\frac{-(1+2 x)(1+6 x)}{2} \\
q(x) & :=-8 x^{2}(1+x) \\
r(x) & :=\frac{1}{16}+x+\frac{3}{2} x^{2}-4 x^{3}-3 x^{4} .
\end{aligned}
$$

$$
z^{4}+p z^{2}+q z+r=\left(z^{2}+\frac{u}{2}\right)^{2}-\left(z^{2}(u-p)-q z+\left(\frac{u^{2}}{4}-r\right)\right) .
$$

We choose $u$ so that the second term in (4.17), a quadratic form in $z$, is a perfect square. This is found by putting the discriminant of the quadratic form in $z$ equal to zero, thereby obtaining a cubic equation in $u$,

$$
u^{3}-p u^{2}-4 r u+\left(4 r p-q^{2}\right)=0 .
$$


Since $p(x), q(x)$, and $r(x)$ are all real valued functions, the polynomial in $u$ must have at least one real solution, which is denoted by $u_{0}(x)$. Hence,

$$
\begin{aligned}
z^{4}+p z^{2}+q z+r= & {\left[z^{2}+z \sqrt{u_{0}-p}+\left(\frac{u_{0}}{2}-\frac{q}{2 \sqrt{u_{0}-p}}\right)\right] } \\
& \times\left[z^{2}-z \sqrt{u_{0}-p}+\left(\frac{u_{0}}{2}+\frac{q}{2 \sqrt{u_{0}-p}}\right)\right] .
\end{aligned}
$$

Thus,

$$
\begin{aligned}
& P_{4}(\lambda)= \\
& \quad\left[\lambda^{2}+\left(\sqrt{u_{0}-p}-(2 x+1)\right) \lambda+\frac{(2 x+1)^{2}}{4}+\frac{u_{0}}{2}-\frac{(2 x+1) \sqrt{u_{0}-p}}{2}-\frac{q}{2 \sqrt{u_{0}-p}}\right] \\
& \times\left[\lambda^{2}-\left(\sqrt{u_{0}-p}+(2 x+1)\right) \lambda+\frac{(2 x+1)^{2}}{4}+\frac{u_{0}}{2}+\frac{(2 x+1) \sqrt{u_{0}-p}}{2}+\frac{q}{2 \sqrt{u_{0}-p}}\right] .
\end{aligned}
$$

The cubic equation in $u$ can be solved by a trigonometric method [2], obtaining the root that is both real and greater than $p(x)$, which is

$$
u_{0}(x)=\frac{-(2 x+1)(6 x+1)+4 \Omega(x) \sqrt{1+16 x+40 x^{2}}}{6}
$$

where

$$
\Omega(x)=\cosh \left[\frac{1}{3} \cosh ^{-1}\left(\frac{1+24 x+156 x^{2}+224 x^{3}+216 x^{4}}{\left(1+16 x+40 x^{2}\right)^{3 / 2}}\right)\right] .
$$

Considering the factorization of $P_{4}(\lambda)$ above, we note that the discriminant of the first quadratic is

$$
\Delta^{-}(x):=\frac{2 q(x)}{\sqrt{u_{0}(x)-p(x)}}-u_{0}(x)-p(x)<0, \quad x>0,
$$

whilst that of the second is

$$
\Delta^{+}(x):=\frac{-2 q(x)}{\sqrt{u_{0}(x)-p(x)}}-u_{0}(x)-p(x)>0, \quad x>0 .
$$

We conclude that for $x>0, P_{4}(\lambda)$ has a complex conjugate pair of roots and two real roots which are identified as $\mu_{1}(x):=e^{t^{-}(x)}$ and $\mu_{2}(x):=e^{t^{+}(x)}$.

Hence, the integral (4.16) becomes

$$
\sigma(x, t)=\frac{1}{\pi t} \int_{\mu_{1}(x)}^{\min \left(\mu, \mu_{2}(x)\right)} \frac{d \lambda}{\lambda \sqrt{\left(\lambda-\mu_{1}(x)\right)\left(\mu_{2}(x)-\lambda\right)\left(\lambda^{2}+\nu(x) \lambda+\xi(x)\right)}}
$$

where $\mu=e^{t}$ and

$$
\begin{gathered}
\nu(x):=\sqrt{u_{0}(x)-p(x)}-(2 x+1)>0, \quad x>0, \\
\xi(x):=\frac{(2 x+1)^{2}}{4}+\frac{u_{0}(x)}{2}-\frac{(2 x+1) \sqrt{u_{0}(x)-p(x)}}{2}-\frac{q(x)}{2 \sqrt{u_{0}(x)-p(x)}}>0, \quad x>0 .
\end{gathered}
$$


We proceed to reduce this integral to a standard form by using the substitution following [3]:

$$
y=\frac{\phi(x)-\lambda}{\lambda-\psi(x)}
$$

which yields

$$
\sigma(x, t)=\frac{1}{\pi t} \int_{\lambda=\mu_{1}}^{\lambda=\min \left(\mu_{2}, \mu\right)} \frac{(\psi(x)-\phi(x))(1+y) d y}{(\phi(x)+\psi(x) y) \sqrt{M(y) N(y)}}
$$

where

$$
\begin{aligned}
& M(y)=\left(\mu_{1}(x)+\mu_{2}(x)\right)(1+y)(\phi(x)+\psi(x) y) \\
& \quad-\mu_{1}(x) \mu_{2}(x)(1+y)^{2}-(\phi(x)+\psi(x) y)^{2} \\
&N(y)=(\phi(x)+\psi(x) y))^{2}+\nu(x)(\phi(x)+\psi(x) y)(1+y)+\xi(x)(1+y)^{2} .
\end{aligned}
$$

We now choose $\phi(x)$ and $\psi(x)$ so that the coefficient of $y$ in both $M(y)$ and $N(y)$ vanishes. This implies

$$
\left(\mu_{1}+\mu_{2}\right)(\phi+\psi)-2 \mu_{1} \mu_{2}-2 \phi \psi=0
$$

and

$$
2 \phi \psi+\nu(\phi+\psi)+2 \xi=0
$$

The solution of these equations gives

$$
\begin{gathered}
\phi+\psi=\frac{2\left(\mu_{1} \mu_{2}-\xi\right)}{\mu_{1}+\mu_{2}+\nu} \\
\phi \psi=-\frac{\xi\left(\mu_{1}+\mu_{2}\right)+\nu \mu_{1} \mu_{2}}{\mu_{1}+\mu_{2}+\nu}<0 .
\end{gathered}
$$

It is easily seen that $\phi$ and $\psi$ are real. With $\phi$ and $\psi$ satisfying these equations we have

$$
\begin{aligned}
M(y) & :=m_{1}+m_{2} y^{2}:=\left(\phi-\mu_{1}\right)\left(\mu_{2}-\phi\right)+\left(\psi-\mu_{1}\right)\left(\mu_{2}-\psi\right) y^{2}, \\
N(y) & :=n_{1}+n_{2} y^{2}:=\left(\phi^{2}+\nu \phi+\xi\right)+\left(\psi^{2}+\nu \psi+\xi\right) y^{2} .
\end{aligned}
$$

Observe that $n_{1}, n_{2}>0$ for $x>0$ since $y^{2}+\nu y+\xi=0$ has no real roots. Further, since the product of $\phi$ and $\psi$ is negative and both $\phi$ and $\psi$ satisfy the same quadratic equation, we are at liberty to choose which root is positive and which is negative. We suppose that $\psi<0$. This means that $m_{2}<0$, consequently $m_{1}>0$ (so that $\left.\phi \in\left(\mu_{1}(x), \mu_{2}(x)\right)\right)$ or else the argument of the square root in the denominator of the integrand is negative. Let us define

Note that

$$
n:=\sqrt{\frac{n_{1}}{n_{2}}}, \quad m:=\sqrt{\frac{m_{1}}{\left|m_{2}\right|}} .
$$

$$
M\left(\frac{\phi-\mu_{1}}{\mu_{1}-\psi}\right)=M\left(\frac{\phi-\mu_{2}}{\mu_{2}-\psi}\right)=0
$$

so

$$
\left(\frac{\phi-\mu_{1,2}}{\mu_{1,2}-\psi}\right)^{2}=m^{2}
$$


Since $\phi \in\left(\mu_{1}, \mu_{2}\right)$ and $\psi<0, x>0$, we see that

$$
\frac{\phi-\mu_{1}}{\mu_{1}-\psi}=m, \quad \text { and } \quad \frac{\phi-\mu_{2}}{\mu_{2}-\psi}=-m
$$

Thus,

$$
\sigma(x, t)=\frac{1}{\pi t} \frac{\left(\frac{\phi}{\psi}-1\right)}{\sqrt{n_{2}\left|m_{2}\right|}} \int_{\max \left(-m, m_{0}\right)}^{m} \frac{(1+y) d y}{\left(\frac{\phi}{\psi}+y\right) \sqrt{\left(n^{2}+y^{2}\right)\left(m^{2}-y^{2}\right)}}
$$

where $m_{0}=\frac{\phi-\mu}{\mu-\psi}$. The integrand is now decomposed into the sum of an odd function and an even function by noticing that

$$
\frac{1+y}{\frac{\phi}{\psi}+y}=1+\frac{\frac{\phi}{\psi}\left(1-\frac{\phi}{\psi}\right)}{\frac{\phi^{2}}{\psi^{2}}-y^{2}}+\frac{y\left(\frac{\phi}{\psi}-1\right)}{\frac{\phi^{2}}{\psi^{2}}-y^{2}}
$$

For $x \in[0, a(t)]$, the interval of integration is $(-m, m)$, thus,

$$
\sigma(x, t)=\frac{2\left(\frac{\phi}{\psi}-1\right)}{\pi t \sqrt{n_{2}\left|m_{2}\right|}} \int_{0}^{m}\left(1+\frac{\frac{\phi}{\psi}\left(1-\frac{\phi}{\psi}\right)}{\frac{\phi^{2}}{\psi^{2}}-y^{2}}\right) \frac{d y}{\sqrt{\left(n^{2}+y^{2}\right)\left(m^{2}-y^{2}\right)}}
$$

From [9], this is

$$
\begin{aligned}
\sigma(x, t)= & \frac{2\left(\frac{\phi}{\psi}-1\right)}{\pi t \sqrt{n_{2}\left|m_{2}\right|}}\left[\frac{n^{2}+\frac{\phi}{\psi}}{\left(n^{2}+\frac{\phi^{2}}{\psi^{2}}\right) \sqrt{m^{2}+n^{2}}} F\left(\frac{\pi}{2}, \frac{m}{\sqrt{m^{2}+n^{2}}}\right)\right. \\
& \left.+\frac{n^{2}\left(1-\frac{\phi}{\psi}\right)}{\frac{\phi}{\psi}\left(n^{2}+\frac{\phi^{2}}{\psi^{2}}\right) \sqrt{m^{2}+n^{2}}} \Pi\left(\frac{\pi}{2}, \frac{m^{2}\left(\frac{\phi^{2}}{\psi^{2}}+n^{2}\right)}{\frac{\phi^{2}}{\psi^{2}}\left(m^{2}+n^{2}\right)}, \frac{m}{\sqrt{m^{2}+n^{2}}}\right)\right]
\end{aligned}
$$

For $x \in[a(t), b(t)]$, there are two distinct cases. Firstly, when $-m<m_{0}<0$ corresponding to $\phi(x)<\mu<\mu_{2}(x)$, then

$$
\sigma(x, t)=\frac{\frac{\phi}{\psi}-1}{\pi t \sqrt{n_{2}\left|m_{2}\right|}}\left[I_{1}+\int_{0}^{-m_{0}}+\int_{0}^{m} \frac{d y}{\sqrt{\left(n^{2}+y^{2}\right)\left(m^{2}-y^{2}\right)}}\left(1+\frac{\frac{\phi}{\psi}\left(1-\frac{\phi}{\psi}\right)}{\frac{\phi^{2}}{\psi^{2}}-y^{2}}\right)\right]
$$

where

$$
I_{1}(x, t)=\int_{m_{0}}^{m} \frac{y\left(\frac{\phi}{\psi}-1\right)}{\frac{\phi^{2}}{\psi^{2}}-y^{2}} \frac{d y}{\sqrt{\left(n^{2}+y^{2}\right)\left(m^{2}-y^{2}\right)}}
$$


Note that $I_{1}$ can be expressed in terms of elementary functions, to be shown later. Again, using [9], we find

$$
\begin{aligned}
\sigma(x, t)= & \frac{\frac{\phi}{\psi}-1}{\pi t \sqrt{n_{2}\left|m_{2}\right|}}\left[I_{1}+\frac{n^{2}+\frac{\phi}{\psi}}{\left(\frac{\phi^{2}}{\psi^{2}}+n^{2}\right) \sqrt{m^{2}+n^{2}}} F\left(\frac{\pi}{2}, \frac{m}{\sqrt{m^{2}+n^{2}}}\right)\right. \\
& +\frac{n^{2}\left(1-\frac{\phi}{\psi}\right)}{\frac{\phi}{\psi}\left(\frac{\phi^{2}}{\psi^{2}}+n^{2}\right) \sqrt{m^{2}+n^{2}}} \Pi\left(\frac{\pi}{2}, \frac{m^{2}\left(\frac{\phi^{2}}{\psi^{2}}+n^{2}\right)}{\frac{\phi^{2}}{\psi^{2}}\left(m^{2}+n^{2}\right)}, \frac{m}{\sqrt{m^{2}+n^{2}}}\right) \\
& +\frac{n^{2}+\frac{\phi}{\psi}}{\left(\frac{\phi^{2}}{\psi^{2}}+n^{2}\right) \sqrt{m^{2}+n^{2}}} F\left(\sin ^{-1}\left(\frac{-m_{0}}{m} \sqrt{\frac{m^{2}+n^{2}}{m_{0}^{2}+n^{2}}}\right), \frac{m}{\sqrt{m^{2}+n^{2}}}\right) \\
& +\frac{n^{2}\left(1-\frac{\phi}{\psi}\right)}{\frac{\phi}{\psi}\left(\frac{\phi^{2}}{\psi^{2}}+n^{2}\right) \sqrt{m^{2}+n^{2}}} \\
& \left.\times \Pi\left(\sin ^{-1}\left(\frac{-m_{0}}{m} \sqrt{\frac{m^{2}+n^{2}}{m_{0}^{2}+n^{2}}}\right), \frac{m^{2}\left(\frac{\phi^{2}}{\psi^{2}}+n^{2}\right)}{\frac{\phi^{2}}{\psi^{2}}\left(m^{2}+n^{2}\right)}, \frac{m}{\sqrt{m^{2}+n^{2}}}\right)\right]
\end{aligned}
$$

The second case is when $0 \leq m_{0}<m$ corresponds to $\mu_{1}(x)<\mu \leq \phi(x)$, then,

$$
\sigma(x, t)=\frac{\frac{\phi}{\psi}-1}{\pi t \sqrt{n_{2}\left|m_{2}\right|}}\left[I_{1}+\int_{m_{0}}^{m}\left(1+\frac{\frac{\phi}{\psi}\left(1-\frac{\phi}{\psi}\right)}{\frac{\phi^{2}}{\psi^{2}}-y^{2}}\right) \frac{d y}{\sqrt{\left(n^{2}+y^{2}\right)\left(m^{2}-y^{2}\right)}}\right]
$$

where $I_{1}$ is as above. By [9], we may write:

$$
\begin{aligned}
\sigma(x, t)= & \frac{\frac{\phi}{\psi}-1}{\pi t \sqrt{n_{2}\left|m_{2}\right|}}\left[I_{1}+\frac{1}{\sqrt{m^{2}+n^{2}}} F\left(\cos ^{-1} \frac{m_{0}}{m}, \frac{m}{\sqrt{m^{2}+n^{2}}}\right)\right. \\
& \left.+\frac{\frac{\phi}{\psi}\left(1-\frac{\phi}{\psi}\right)}{\left(\frac{\phi^{2}}{\psi^{2}}-m^{2}\right) \sqrt{m^{2}+n^{2}}} \Pi\left(\cos ^{-1} \frac{m_{0}}{m}, \frac{m^{2}}{m^{2}-\frac{\phi^{2}}{\psi^{2}}}, \frac{m}{\sqrt{m^{2}+n^{2}}}\right)\right] .
\end{aligned}
$$

In order to compute $I_{1}$, it is necessary to first indicate the range of the various parameters appearing in (4.33). Recalling the substitution

$$
y(\lambda):=\frac{\phi-\lambda}{\lambda-\psi}
$$

we deduce that in the domain of our problem, $t>0$ and $x>0$,

$$
\left|\frac{\phi}{\psi}\right|>m>\left|m_{0}\right| \text {. }
$$

This provides sufficient information to specify $I_{1}$ precisely, which by means of elementary integration is found to be

$$
\begin{aligned}
I_{1}(x)= & \frac{\phi / \psi-1}{2 \sqrt{\left[n^{2}+(\phi / \psi)^{2}\right]\left[(\phi / \psi)^{2}-m^{2}\right]}} \\
& \times\left[\frac{\pi}{2}+\tan ^{-1} \frac{2 m^{2} n^{2}+\left(m^{2}-n^{2}\right)\left[(\phi / \psi)^{2}+m_{0}^{2}\right]-2(\phi / \psi)^{2} m_{0}^{2}}{2 \sqrt{\left[(\phi / \psi)^{2}-m^{2}\right]\left[(\phi / \psi)^{2}+n^{2}\right]\left(n^{2}+m_{0}^{2}\right)\left(m^{2}-m_{0}^{2}\right)}}\right] .
\end{aligned}
$$

The zero density for both cases, $\gamma=$ constant and $\gamma=n$, are given in Figures 3 and 4 , respectively. 


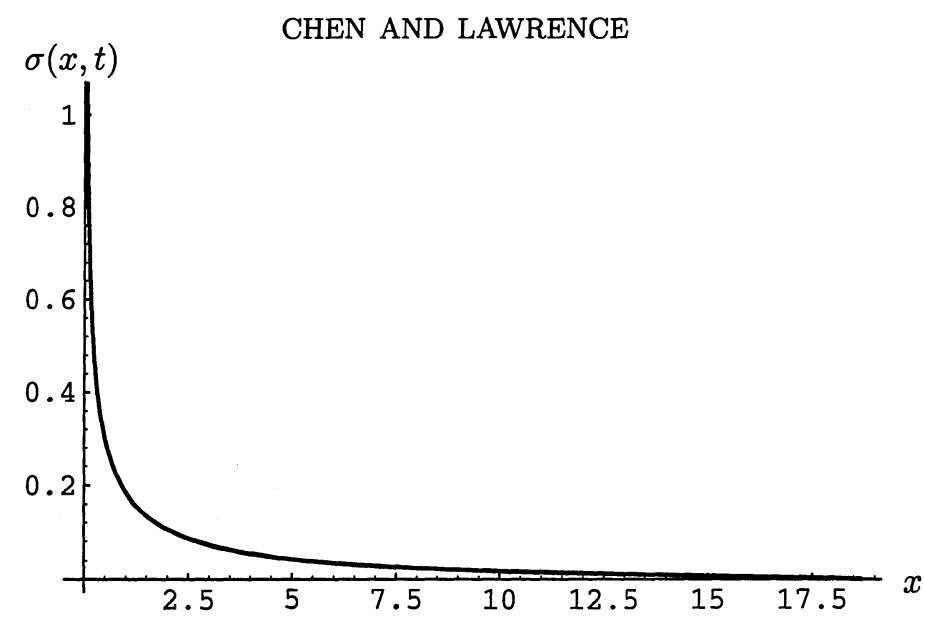

FIgURE 3. Zero density of the $q^{-1}$-Laguerre polynomials where the parameter $\gamma$ is assumed to be constant. Plotted here is the density for $t=1$.

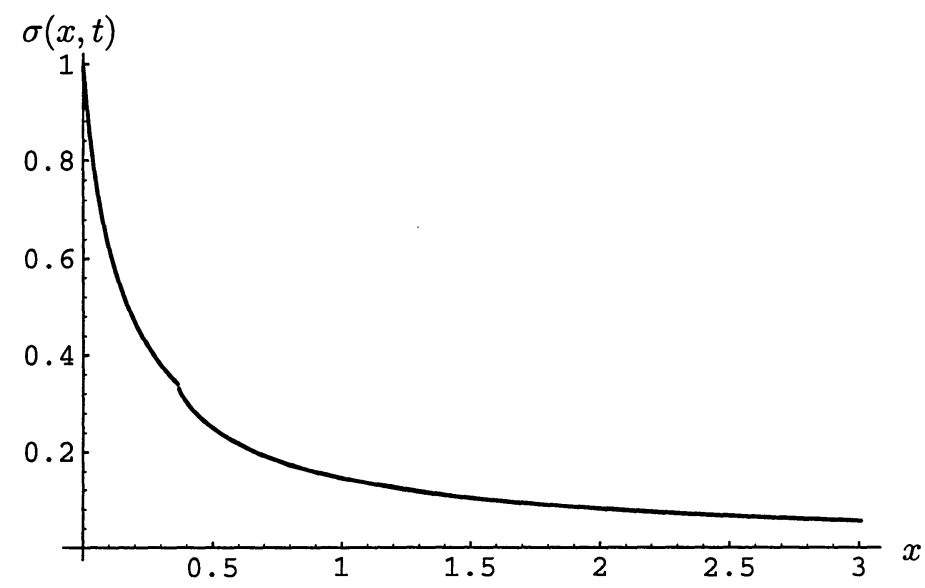

FIGURE 4. Asymptotic zero density for the $q^{-1}$-Laguerre polynomials where $\gamma=n$ and $t=1$. That the plot terminates at $x \approx 3$ does not indicate that the density terminates here since $b(t) \gg 3$. The scale selected enables us to see the discontinuity in the slope of the density. However, near $b(t)$, the density vanishes like a square root. Near 0 , the density diverges like $1 / \sqrt{x}$. This is because the left-end point, namely 0 , is fixed. These features are general. Note the similarity of Figure 3 and this figure.

\section{A constructed set of orthogonal polynomials}

This example clearly illustrates how elliptic functions may occur in the asymptotic zero density. Consider the case:

$$
\begin{aligned}
& a(t):=\mu t, \quad \mu>0, \\
& b(t):=t(\varrho-t), \quad \varrho>0 .
\end{aligned}
$$


To ensure that $b(t)>a(t)$ in $t>0$, we require $\varrho>\mu$ and $t \in[0, \varrho-\mu]$. The density reads

$$
\sigma(x, t)=\frac{1}{\pi t} \int_{t^{-}}^{\min \left(t, t^{+}\right)} \frac{d s}{\sqrt{(b(s)-x)(x-a(s))}}
$$

where $b\left(t^{-}\right)=a\left(t^{+}\right)=x$ if $x<\mu(\varrho-\mu)$ and $b\left(t^{-}\right)=b\left(t^{+}\right)=x$ if $x>\mu(\varrho-\mu)$.

The roots of the quadratic form in the denominator are

$$
s_{1,2}(x)=\frac{\varrho}{2} \pm \sqrt{\frac{\varrho^{2}}{4}-x}
$$

where $s_{1} \leq s_{2}$. Note that in all possible cases, $0 \leq x \leq \frac{\varrho^{2}}{4}$, so that all roots of the cubic equation,

$$
\left(s-\frac{x}{\mu}\right)\left(s^{2}-\varrho s+x\right)=0,
$$

are always real and positive.

Case A: $0<\mu<\varrho / 2 ; 0<t \leq \mu$. When $0 \leq x \leq a(t)$, so that $s_{1}<\frac{x}{\mu}<t<s_{2}$, then

$$
\begin{aligned}
\sigma(x, t) & =\frac{1}{\pi t \sqrt{\mu}} \int_{s_{1}}^{\frac{x}{\mu}} \frac{d s}{\sqrt{\left(s-s_{1}\right)\left(s_{2}-s\right)\left(\frac{x}{\mu}-s\right)}} \\
& =\frac{2}{\pi t \sqrt{\mu\left(s_{2}-s_{1}\right)}} F\left(\frac{\pi}{2}, \sqrt{\frac{\frac{x}{\mu}-s_{1}}{s_{2}-s_{1}}}\right) .
\end{aligned}
$$

When $a(t)<x \leq b(t)$, see Figure 5 , so that $s_{1}<t<\frac{x}{\mu}<s_{2}$, then

$$
\begin{aligned}
\sigma(x, t) & =\frac{1}{\pi t \sqrt{\mu}} \int_{s_{1}}^{t} \frac{d s}{\sqrt{\left(s-s_{1}\right)\left(s_{2}-s\right)\left(\frac{x}{\mu}-s\right)}} \\
& =\frac{2}{\pi t \sqrt{\mu\left(s_{2}-s_{1}\right)}} F\left(\sin ^{-1}\left(\sqrt{\frac{t-s_{1}}{\frac{x}{\mu}-s_{1}}}\right), \sqrt{\frac{\frac{x}{\mu}-s_{1}}{s_{2}-s_{1}}}\right) .
\end{aligned}
$$

Case B: $0<\mu<\varrho / 2 ; \mu<t \leq \varrho / 2$. When $0 \leq x \leq a(t)$, so that $s_{1}<\frac{x}{\mu}<t<s_{2}$, then

$$
\begin{aligned}
\sigma(x, t) & =\frac{1}{\pi t \sqrt{\mu}} \int_{s_{1}}^{\frac{x}{\mu}} \frac{d s}{\sqrt{\left(s-s_{1}\right)\left(s_{2}-s\right)\left(\frac{x}{\mu}-s\right)}} \\
& =\frac{2}{\pi t \sqrt{\mu\left(s_{2}-s_{1}\right)}} F\left(\frac{\pi}{2}, \sqrt{\frac{\frac{x}{\mu}-s_{1}}{s_{2}-s_{1}}}\right) .
\end{aligned}
$$

When $a(t)<x \leq \mu(\varrho-\mu)$, see Figure 5 , so that $s_{1}<t<\frac{x}{\mu}<s_{2}$, then

$$
\begin{aligned}
\sigma(x, t) & =\frac{1}{\pi t \sqrt{\mu}} \int_{s_{1}}^{t} \frac{d s}{\sqrt{\left(s-s_{1}\right)\left(s_{2}-s\right)\left(\frac{x}{\mu}-s\right)}} \\
& =\frac{2}{\pi t \sqrt{\mu\left(s_{2}-s_{1}\right)}} F\left(\sin ^{-1}\left(\sqrt{\frac{t-s_{1}}{\frac{x}{\mu}-s_{1}}}\right), \sqrt{\frac{\frac{x}{\mu}-s_{1}}{s_{2}-s_{1}}}\right) .
\end{aligned}
$$




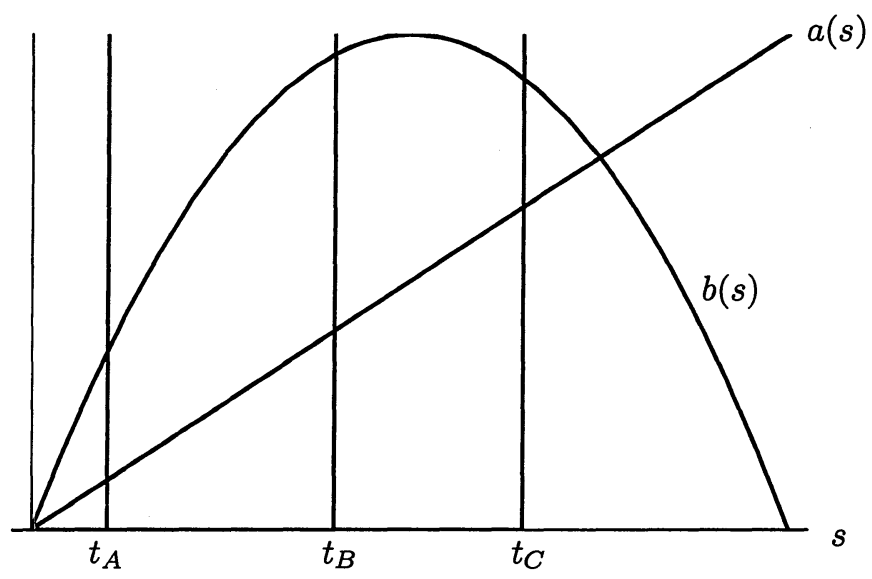

FIgURE 5. Shown here is the typical behavior of $a(s)$ and $b(s)$ in cases $\mathrm{A}, \mathrm{B}$, and $\mathrm{C}$ of the constructed example. Also indicated are possible $t$ values corresponding to each of these cases, respectively.

Finally see Figure 5, for $\mu(\varrho-\mu)<x \leq b(t)$, where $s_{1}<t<s_{2}<\frac{x}{\mu}$, then

$$
\begin{aligned}
\sigma(x, t) & =\frac{1}{\pi t \sqrt{\mu}} \int_{s_{1}}^{t} \frac{d s}{\sqrt{\left(s-s_{1}\right)\left(s_{2}-s\right)\left(\frac{x}{\mu}-s\right)}} \\
& =\frac{2}{\pi t \sqrt{x-\mu s_{1}}} F\left(\sin ^{-1}\left(\sqrt{\frac{t-s_{1}}{s_{2}-s_{1}}}\right), \sqrt{\frac{s_{2}-s_{1}}{\frac{x}{\mu}-s_{1}}}\right) .
\end{aligned}
$$

Case C: $0<\mu<\varrho / 2 ; \varrho / 2<t \leq \varrho-\mu$. For $x \in[0, b(t)]$, see Figure 5 , the expressions for the density are identical to Case B. For $b(t)<x \leq \varrho^{2} / 4$ where $s_{1}<s_{2}<t<\frac{x}{\mu}$, then

$$
\begin{aligned}
\sigma(x, t) & =\frac{1}{\pi t \sqrt{\mu}} \int_{s_{1}}^{s_{2}} \frac{d s}{\sqrt{\left(s-s_{1}\right)\left(s_{2}-s\right)\left(\frac{x}{\mu}-s\right)}} \\
& =\frac{2}{\pi t \sqrt{x-\mu s_{1}}} F\left(\frac{\pi}{2}, \sqrt{\frac{s_{2}-s_{1}}{\frac{x}{\mu}-s_{1}}}\right) .
\end{aligned}
$$

Case D: $\varrho / 2 \leq \mu<\varrho ; 0<t \leq \varrho-\mu$. Note we always have $s_{1}<x / \mu<s_{2}$. When $0 \leq x \leq a(t)$, then

$$
\begin{aligned}
\sigma(x, t) & =\frac{1}{\pi t \sqrt{\mu}} \int_{s_{1}}^{\frac{x}{\mu}} \frac{d s}{\sqrt{\left(s-s_{1}\right)\left(s_{2}-s\right)\left(\frac{x}{\mu}-s\right)}} \\
& =\frac{2}{\pi t \sqrt{\mu\left(s_{2}-s_{1}\right)}} F\left(\frac{\pi}{2}, \sqrt{\frac{\frac{x}{\mu}-s_{1}}{s_{2}-s_{1}}}\right) .
\end{aligned}
$$




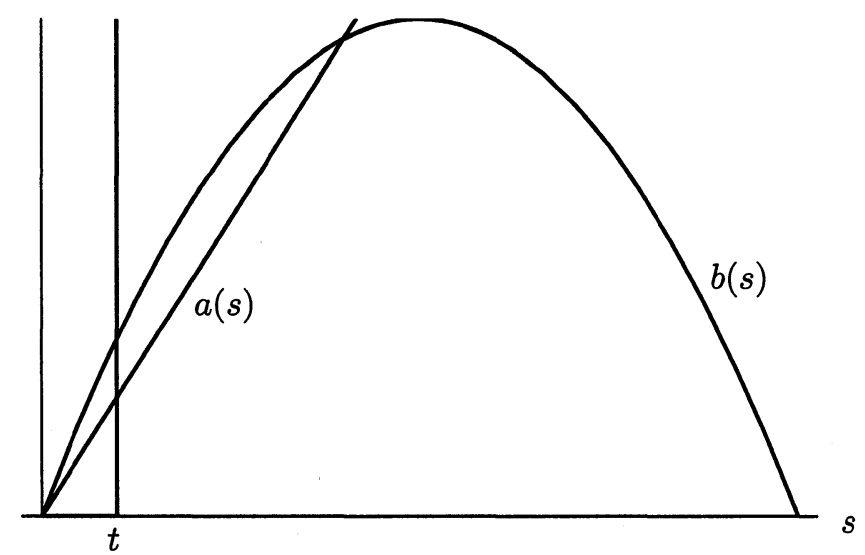

FIGURE 6. Shown here is the typical behavior of $a(s)$ and $b(s)$ in case $\mathrm{D}$ of the constructed example. Also indicated is a possible $t$ value corresponding to this case.

When $a(t)<x \leq b(t)$, see Figure 6 , we find

$$
\begin{aligned}
\sigma(x, t) & =\frac{1}{\pi t \sqrt{\mu}} \int_{s_{1}}^{t} \frac{d s}{\sqrt{\left(s-s_{1}\right)\left(s_{2}-s\right)\left(\frac{x}{\mu}-s\right)}} \\
& =\frac{2}{\pi t \sqrt{\mu\left(s_{2}-s_{1}\right)}} F\left(\sin ^{-1}\left(\sqrt{\frac{t-s_{1}}{\frac{x}{\mu}-s_{1}}}\right), \sqrt{\frac{\frac{x}{\mu}-s_{1}}{s_{2}-s_{1}}}\right) .
\end{aligned}
$$

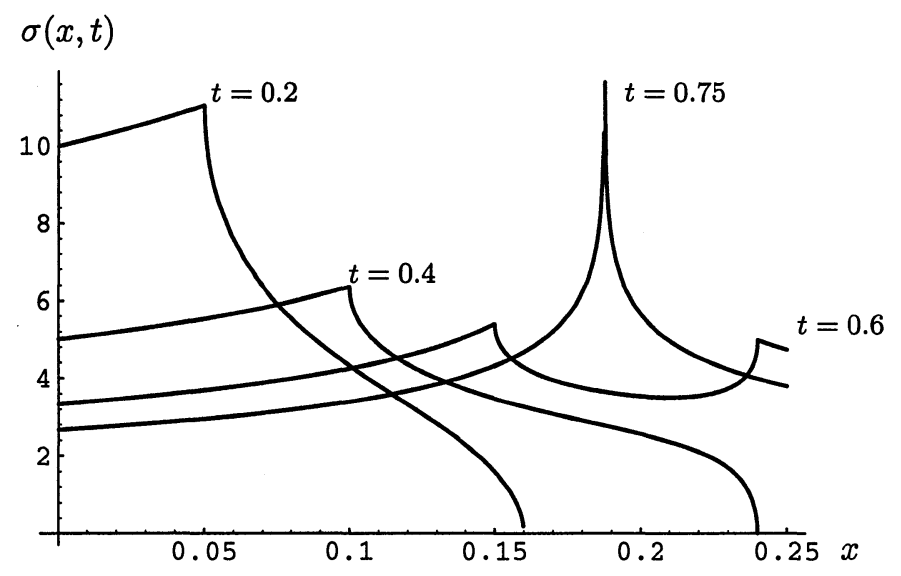

FIGURE 7. Zero density plots for various $t$ values corresponding to cases $\mathrm{A}, \mathrm{B}$, and $\mathrm{C}$ where $\mu<\varrho / 2$. Shown here are the densities when $\varrho=1, \mu=1 / 4$, and $t=0.2,0.4,0.6,0.75$.

Observe that in Figures 7 and 8, which graph $\sigma$ for the above cases, the densities exhibit possible discontinuities in the derivative. We now discuss some of the features observed in the densities generated in the construction above. 


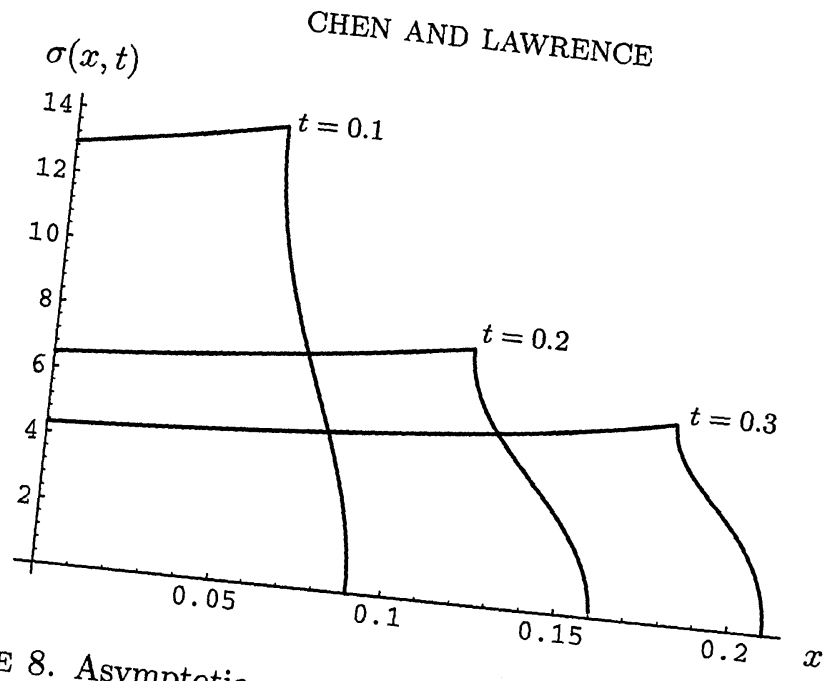

FIGURE 8. Asymptotic zero density plots for various $t$ values, for a
case $D$ example, where $\mu>0$. Shom he are the densties when

In all cases, if we allow

where

$$
\begin{aligned}
& \sigma(x, t) \sim \frac{2}{\pi t \sqrt{\mu\left(s_{2}(\mu t)-s_{1}(\mu t)\right)}} \\
& \quad \times\left[F\left(\frac{\pi}{2}, k(\mu t)\right)-\frac{1}{2 \sqrt{\mu\left(t-s_{1}(\mu t)\right)\left(1-k^{2}(\mu t)\right)}} \sqrt{\delta}\right]+\mathrm{O}(\delta)
\end{aligned}
$$

$$
k(x):=\sqrt{\frac{x / \mu-s_{1}(x)}{s_{2}(x)-s_{1}(x)}} .
$$
Repeating a similar analysis with $\delta \rightarrow-\delta$, we see that the slope does not diverge as
$x \rightarrow \mu t-0$.
If $t<\varrho / 2$, we note that $\sigma(t(\varrho-t), t)=0$. In of $x=t(\varrho-t)-\delta$, we find $\sigma(t(\varrho-t), t)=0$. In cases $A$ and $D$, making the substitution

In case $B$, this $\frac{2 \pi b s t i t u t(\varrho-2 t) \sqrt{\mu+t(\varrho-t)-\frac{\mu \varrho}{2}}}{\pi \delta}+O\left(\delta^{\frac{3}{2}}\right)$

$$
\sigma(x, t) \sim \frac{2}{\pi t(\varrho-2 t) \sqrt{\mu+t(\varrho-t)-\frac{\mu \varrho}{2}}} \sqrt{\delta}+\mathrm{O}\left(\delta^{\frac{3}{2}}\right) .
$$

$$
\sigma(x, t) \sim \frac{2}{\pi t(\varrho-2 t) \sqrt{t(\varrho-t)-\mu t}} \sqrt{\delta}+O\left(\delta^{\frac{3}{2}}\right) . \quad \text { of the density as }
$$


Thus, we see that the gradient diverges as $x \rightarrow t(\varrho-t)-0$, to $-\infty$, for each case where $t<\varrho / 2$.

However, if $t>\varrho / 2$, as it is in case $\mathrm{C}$, by applying the above substitution, we find

$$
\begin{aligned}
\sigma(x, t) \sim & \frac{2}{\pi t \sqrt{t(\varrho-t)-\mu s_{1}(t(\varrho-t))}}\left[F\left(\frac{\pi}{2}, k(t(\varrho-t))\right)\right. \\
& \left.\quad-\frac{1}{2\left(s_{2}(t(\varrho-t))-s_{1}(t(\varrho-t))\right) \sqrt{1-k^{2}(t(\varrho-t))}} \sqrt{\delta}\right]+O(\delta) .
\end{aligned}
$$

From this expression, it can be seen that as $x \rightarrow t(\varrho-t)-0$, the gradient again diverges, but to $+\infty$. Again by letting $\delta \rightarrow-\delta$, the asymptotic analysis shows that for $t>\varrho / 2$, the slope does not diverge for $x \rightarrow t(\varrho-t)+0$.

We now mention a special case where $\mu<\varrho / 2$ and $t=\varrho-\mu$. The density is seen to diverge at $x=\mu(\varrho-\mu)$. In order to understand the nature of the divergence, we use the expansion [9]:

$$
F\left(\frac{\pi}{2}, k\right) \sim \ln \frac{4}{\sqrt{1-k^{2}}}+\left(\frac{1}{2}\right)^{2}\left(\ln \frac{4}{\sqrt{1-k^{2}}}-1\right)\left(1-k^{2}\right)+\text { H.O.T. }, \quad k \rightarrow 1
$$

If $x=\mu(\varrho-\mu) \pm \delta$ where $\delta \rightarrow 0$, the density has the form:

$$
\sigma(x, t) \sim \frac{1}{\pi t \sqrt{\mu(\varrho-2 \mu)}} \ln \frac{1}{\delta}+O(1) .
$$

As such, we can see that the gradient at this point behaves like $(\mu(\varrho-\mu)-x)^{-1}$. The zero density for cases A, B, and C are shown in Figure 7 and case D in Figure 8.

\section{References}

1. N. I. Akhiezer, The Classical Moment Problem and Some Related Questions in Analysis, Oliver and Boyd, Edinburgh, 1965.

2. G. Birkhoff and S. MacLane, A Survey of Modern Algebra, (4th edition), Macmillan, New York, 1977.

3. A. Cayley, An Elementary Treatise on Elliptic Functions, Dover, New York, 1956.

4. Y. Chen and M. E. H. Ismail, Thermodynamic relations of the Hermitian matrix ensembles, J. Phys. A. 30 (1997), 6633-6654, and the references therein give an overview of the Coulomb fluid approximation.

5. Y. Chen, M. E. H. Ismail, and K. A. Muttalib, Metallic and insulating behaviour in an exactly solvable random matrix model, J. Phys. Condensed Matter 5 (1993), 177-190.

6. Y. Chen and N. Lawrence, On the linear statistics of Hermitian matrix ensembles, J. Phys. A. 31 (1998), 1141-1152.

7. F. J. Dyson, Statistical theory of energy levels of complex systems, I, II, III, J. Math. Phys. 3 (1962), 140-156, 157-165, 166-175.

8. F. D. Gakhov, Boundary Value Problems, Dover, New York, 1990.

9. I. S. Gradshteyn and I. M. Ryzhik, Table of Integals, Series and Products, Academic Press, 1965. 
10. A. B. Kuijlaars and W. Van Assche, The asymptotic zero distribution of orthogonal polynomials with varying recurrence coefficients, (to be published).

11. E. B. Saff and V. Totik, Logarithmic Potential with External Fields, Springer, New York, 1997.

Department of Mathematics, Imperial College, 180 Queen's Gate, London, SW7 2BZ, UNITED KINGDOM 